hep-ph/0102xxx

OUTP-0107P

JLAB-THY-01-01

6 February 2001

\title{
The origins of quark-hadron duality: How does the square of the sum become the sum of the squares?
}

\author{
Frank E. Close \\ Dept of Theoretical Physics \\ University of Oxford, Keble Rd., Oxford, OX1 3NP, England \\ Nathan Isgur!.! \\ Jefferson $L a b$ \\ 12000 Jefferson Avenue, Newport News, Virginia 23606, USA
}

\begin{abstract}
Bloom-Gilman duality demonstrates empirically that the electroproduction of $N^{*}$ s at low momentum transfers averages smoothly around the scaling curve measured at large momentum transfers. The latter is proportional to the sum of the squares of the constituent charges whereas the former involves the coherent excitation of resonances and is driven by the square of summed constituent charges. We determine the minimal necessary conditions for this equality to be realised so that duality can occur and consider the implications for a range of processes that may be studied soon at CEBAF.
\end{abstract}

\footnotetext{
1e-mail: F.E.Close@rl.ac.uk

${ }^{2}$ e-mail: isgur@jlab.org
} 
When protons are probed by electron beams at energies of tens or hundreds of $\mathrm{GeV}$, and at correspondingly large momentum transfers $\left(Q^{2}\right)$, the scattering probability (summarised in the structure function $F_{2}\left(W^{2}, Q^{2}\right)$ where $W$ is the mass of the hadronic system) is rather simple. It exhibits the well known property of scale invariance where $F_{2} \sim F_{2}\left(W^{2} / Q^{2}\right)$, with small corrections that are well understood from perturbative QCD. Furthermore, the magnitude of $F_{2}\left(W^{2} / Q^{2}\right)$ is proportional to the sum of the squares of the (quark and antiquark) constituent charges.

Before the advent of QCD, Bloom and Gilman discovered [i] an empirical property of the data, namely that the electroproduction of $N^{*}$ 's at lower energies and momentum transfers averages smoothly around the scaling curve measured at large momentum transfers. During the subsequent three decades, and especially following the advent of quantum chromodynamics, this enigma has received considerable theoretical attention [2] [- The literature has primarily focussed on understanding how the $Q^{2}$ dependence of resonance excitation can conspire to mimic the $x^{\prime} \sim \frac{Q^{2}}{W^{2}+Q^{2}}$ dependence of the deep inelastic data.

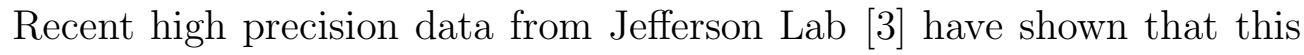
duality is observed for both proton and neutron targets, at least for spin averaged scattering, and that for the proton it occurs locally, i.e., resonance by resonance. This has sparked a renewed interest in the origin of low energy duality, including a recent analysis in the context of a large- $N_{c}$-based relativistic quark model [i] confined struck quark to behave as though it were free, but remarked in passing on the additional conditions on quark charges and dynamics required for duality to be realized locally. These data and the work of Ref. 郅 have led us to focus in this paper on the latter issue, namely the circumstances whereby $F_{2}\left(x^{\prime}\right)$ - whose magnitude is in proportion to the sum of the squares of the (quark and antiquark) constituent charges - can in general match with the excitation of individual resonances which is driven by the coherently summed square of constituent charges.

In this note we draw attention to the necessary conditions for this duality to occur in general. We illustrate the essential physical principle in a simple pedagogical model of a system of two spinless electrically charged consitu- 
tents. This is then generalised to the more realistic case of three spinning quarks. Implications for both spin-dependent and spin-independent structure functions for proton and neutron targets will be displayed, local deviations from duality are predicted, and the possibility that there could be "precocious" factorisation in semi-inclusive hadroproduction is discussed. These ideas promise a lively program of experimental investigation for CEBAF.

\section{A Simple Model}

Consider a composite state made of two equal mass scalars, "quarks" $q_{1}, q_{2}$ with charges $e_{1}, e_{2}$ respectively at positions $\vec{r}_{1,2}$. The ground state wavefunction is $\psi_{0}(\vec{r})$, where $\vec{r}_{1,2}=\vec{R} \pm \vec{r} / 2$ defines the centre of mass and internal spatial degrees of freedom. A photon of momentum $\vec{q}$ is absorbed with an amplitude proportional to $\Sigma_{i} e_{i} \exp \left(i \vec{q} \cdot \vec{r}_{i}\right)$, which excites a "resonant" state with angular momentum L, described by the wavefunction $\psi_{L}(\vec{r})$.

Focussing on the internal coordinate $\vec{r}$, the transition amplitude is proportional to

$$
\left(e_{1}+e_{2}\right)(\exp (i \vec{q} \cdot \vec{r} / 2)+\exp (-i \vec{q} \cdot \vec{r} / 2))+\left(e_{1}-e_{2}\right)(\exp (i \vec{q} \cdot \vec{r} / 2)-\exp (-i \vec{q} \cdot \vec{r} / 2))
$$

The expansion, $\exp (i q z / 2)=\Sigma_{L} i^{L} P_{L}(\cos \theta) j_{L}(q r / 2)(2 L+1)$, projects out the even and odd partial waves such that the amplitude is proportional to

$$
M \sim \int d r r^{2} \psi_{L}^{*}(r) \psi_{0}(r) j_{L}(q r / 2)\left[\left(e_{1}+e_{2}\right) \delta_{L=\text { even }}+\left(e_{1}-e_{2}\right) \delta_{L=o d d}\right] .
$$

The resulting structure function, summed over resonance excitations, will have the form

$$
F(q) \sim \sum_{n=0}^{\infty}\left[F_{2 n}(q)\left(e_{1}+e_{2}\right)^{2}+F_{2 n+1}(q)\left(e_{1}-e_{2}\right)^{2}\right]
$$

which in general will be proportional to $e_{1}^{2}+e_{2}^{2}$ only if the odd and even $L$ states sum to equal strengths.

We now derive the circumstances under which this can occur. Consider the $u u,(u d \pm d u) / \sqrt{2}$ and $d d$ composite systems subject to the following 
process: $W^{+}+(d d) \rightarrow(u d \pm d u) / \sqrt{2} \rightarrow W^{-}+(u u)$. The overall Bose symmetry of the system will constrain the $u d+(-) d u$ intermediate states to have $L=2 n(L=2 n+1)$ respectively. Following analogous steps to those above, the amplitude will have the form

$$
A\left(W^{+}(q)+(d d) \rightarrow W^{-}(q)+(u u)\right) \sim \sum_{n=0}^{\infty}\left[F_{2 n}(q)-F_{2 n+1}(q)\right]
$$

In this case the $t$-channel involves transfer of exotic quantum numbers (charge 2, corresponding to constituents $u u \bar{d} \bar{d}$ ) and in the absence of such states, the amplitude must vanish according to the theory of hadronic duality [5]

$$
\sum_{n=0}^{\infty} F_{2 n}(q) \equiv \sum_{n=0}^{\infty} F_{2 n+1}(q)
$$

which with Eqn.(ㄴiㄴ) leads to

$$
F(q) \sim \sum_{n=0}^{\infty} F_{n}(q)\left(e_{1}^{2}+e_{2}^{2}\right)
$$

whereby the square of the sum has become the sum of the squares.

This simple example exposes the physics rather clearly. The excitation amplitudes to resonance states contain both diagonal $\left(e_{1}^{2}+e_{2}^{2}\right)$ and higher twist terms $\left( \pm 2 e_{1} e_{2}\right)$ in the flavour basis. The former set add constructively for any $L$ and the sum over the complete set of states can now logically give the deep inelastic curve [2]; the latter enter with opposite phases for even and odd $L$ and destructively interfere. The critical feature that this exposes is that at least one complete set of resonances of each symmetry-type has been summed over.

In a non-relativistic SHO model it is possible to see how the above conspiracy arises. The contribution to $F(q)$ from the $n(\equiv L+2 k)$ set of degenerate levels ( $k$ being the radial and $L$ the orbital quantum number) 
is $F_{n}(q) \sim(n !)^{-1}\left(q^{2} R^{2}\right)^{n} e^{-q^{2} R^{2}}$ from which one can immediately see that $\Sigma_{n} F_{n}(q)=1$. It is interesting to note that any individual contribution, $F_{n}$, reaches its maximum value when $q^{2} R^{2}=n$, at which point $F_{n}=F_{n-1}$. This coincidence is true for all juxtaposed partial waves at their peaks, which gives a rapid approach to the equality of $F_{\text {odd }}$ and $F_{\text {even }}$. Analytically in the SHO one finds

$$
F(q) \sim\left(e_{1}^{2}+e_{2}^{2}\right)+2 e_{1} e_{2} e^{-4 q^{2} R^{2}}
$$

whereby the coherent (duality-violating) terms vanish like the fourth power of the elastic form factor.

\section{SHO Quark Model}

This pedagogical example contains all the essential physics needed to understand the more realistic case of the harmonic oscillator quark model. The example of spinless constituents above involved only electric multipoles, whereas introduction of spin involves both electric and magnetic multipole contributions. The symmetric and antisymmetric states generalise, respectively, to the $\mathbf{5 6}$ and $\mathbf{7 0}$ representations of $S U(6)_{\text {flavor-spin }}$. The destructive interference in the $s$-channel sum is as before and is now correlated with the overall Fermi antisymmetry of the $S U(6) \times S U(3)_{\text {color }} \times \psi(r)$ wavefunctions. The quark-parton model scaling curves proportional to $\Sigma_{i} e_{i}^{2}$ then obtain if $F_{1}(\mathbf{5 6}) \equiv F_{1}(\mathbf{7 0})$ and if the interaction of the photon with the magnetic moment of the quarks dominates.

This numerology was demonstrated long ago in Ref. [6], which exhibited the decomposition of the structure functions for $\gamma+N \rightarrow$ hadrons when the $\gamma$ and $N$ spins are antiparallel $\left(\sigma_{1 / 2}\right)$ or parallel $\left(\sigma_{3 / 2}\right)$ (i.e., when the net spin projection along the initial photon direction is $1 / 2 ; 3 / 2)$. The relative strengths of the supermultiplets for the case where magnetic interactions dominate are listed in Table 1 from which $g_{1}^{p, n} \sim \sigma_{1 / 2}-\sigma_{3 / 2}$ and $F_{1}^{p, n} \sim \sigma_{1 / 2}+\sigma_{3 / 2}$ can be constructed. The familiar leading twist ratios are obtained immediately from an equal weighting of the $\mathbf{5 6}$ and $\mathbf{7 0}$ contributions: $F^{n} / F^{p}=2 / 3 ; A^{p} \equiv g_{1}^{p} / F_{1}^{p}=5 / 9 ; A^{n}=0$. 
Table 1: Relative Photoproduction Strengths in the Quark Model

\begin{tabular}{lcccc}
\hline$S U(6):$ & $\sigma_{1 / 2}^{p}$ & $\sigma_{3 / 2}^{p}$ & $\sigma_{1 / 2}^{n}$ & $\sigma_{3 / 2}^{n}$ \\
\hline $\mathbf{5 6}$ & 11 & 6 & 6 & 6 \\
$\mathbf{7 0}$ & 10 & 0 & 3 & 3 \\
\hline
\end{tabular}

These results imply that for $F_{1}^{p, n}$ and $g_{1}^{p}$ duality will not be realised unless the 56 and 70 states $\left(N, \Delta(1236)\right.$ and the negative parity $N^{*}, \Delta^{*}$ with $W \leq 1.8$ $\mathrm{GeV}$ ) have been integrated over. However, for $g_{1}^{n}$ there is the tantalising possibility that duality may be more localised as the $\mathbf{5 6}$ alone, consisting of $N, \Delta(1236)$ already satisfies $\int d W g_{1}^{n}(W, q)=0$ in this idealised model, and the dominance of magnetic interactions is also realised for the neutron target. In reality $\mathrm{SU}(6)$ is broken, in particular by the differing masses of the $N$ and $\Delta$ due to higher twist one-gluon-exchange hyperfine effects in QCD. Hence it will be interesting to investigate these general ideas in explicit quark models to predict the detailed approach to duality in a world where $\mathrm{SU}(6)$ is broken. Some qualitative ideas on the $x \rightarrow 1$ dependence of $F^{n} / F^{p}$ and $A^{n, p}$ already exist in the literature leading twist results relate to the symmetries of the excited hadronic states, explicit calculations summing over the above resonances and incorporating $\mathrm{SU}(6)$ breaking in QCD can now be developed and their implications for CEBAF in particular explored.

When we break Table 1 into its $S U(3) \times S U(2)$ content, we see that for the proton duality may be satisfied by $W \leq 1.6 \mathrm{GeV}$. This is because the $\mathbf{7 0} ;{ }^{4} \mathbf{8}$ and $\mathbf{7 0} ;{ }^{\mathbf{2}} \mathbf{1 0}$, which are at $\sim 1.7 \mathrm{GeV}$, make negligible contributions (Table 2). For neutron targets, by contrast, one must include the $\mathbf{7 0} ;{ }^{4} \mathbf{8}$, which necessitates integrating up to $1.8 \mathrm{GeV}$. This region above $1.7 \mathrm{GeV}$ also contains 56 at $N=2$ in the harmonic oscillator. Thus we anticipate systematic deviations from local duality, in accord with the new data from Ref. [3ij. These data showed that the $S_{11}(1530)$ region $\left(\mathbf{7 0} ;{ }^{2} \mathbf{8}\right)$ and the $F_{15}(1680)(\mathbf{5 6} ; \mathbf{2} \mathbf{8})$ are enhanced relative to the deep inelastic scaling curve for proton targets. As Table 2 shows, the $\mathbf{7 0}$ contribution for a proton target is concentrated in the $\mathbf{7 0}^{\mathbf{2}} \mathbf{8}$, hence the enhancement seen in Ref. [3] 
Table 2: Relative Photoproduction Strengths of 56, $\mathbf{0}^{+}$and $\mathbf{7 0 , \mathbf { 1 } ^ { - }}$ Multiplets

\begin{tabular}{lccccccc}
\hline$S U(6):$ & {$\left[\mathbf{5 6}, \mathbf{0}^{+}\right]^{\mathbf{2}} \mathbf{8}$} & {$\left[\mathbf{5 6}, \mathbf{0}^{+}\right]^{\mathbf{4}} \mathbf{1 0}$} & {$\left[\mathbf{7 0}, \mathbf{1}^{-}\right]^{\mathbf{2}} \mathbf{8}$} & {$\left[\mathbf{7 0}, \mathbf{1}^{-}\right]^{\mathbf{4}} \mathbf{8}$} & {$\left[\mathbf{7 0}, \mathbf{1}^{-}\right]^{\mathbf{2}} \mathbf{1 0}$} & total \\
\hline$F_{1}^{p}$ & 9 & 8 & 9 & 0 & 1 & 27 \\
$F_{1}^{n}$ & 4 & 8 & 1 & 4 & 1 & 18 \\
$g_{1}^{p}$ & 9 & -4 & 9 & 0 & 1 & 15 \\
$g_{1}^{n}$ & 4 & -4 & 1 & -2 & 1 & 0 \\
\hline
\end{tabular}

In contrast to the proton case, this table predicts that for neutron targets, the $S_{11}(1530)$ region $\left(\left[\mathbf{7 0}, \mathbf{1}^{-}\right]^{\mathbf{2}} \mathbf{8}\right)$ will fall below the scaling curve. The third resonance region, containing $\left[\mathbf{7 0}, \mathbf{1}^{-}\right]^{\mathbf{4}} \mathbf{8}$ as well as $\left[\mathbf{5 6}, \mathbf{2}^{+}\right]^{\mathbf{2}} \mathbf{8}$ and $\left[\mathbf{5 6}, \mathbf{2}^{+}\right]^{\mathbf{4}} \mathbf{1 0}$, is expected to be locally enhanced over the scaling curve for both proton and neutron targets. Note that to order $q^{2}$ the $\left[\mathbf{5 6}, \mathbf{0}^{+}\right]$and $\left[\mathbf{7 0}, \mathbf{1}^{-}\right]$multiplets are sufficient to realise duality. Formally the analyis can be extended to higher $q^{2}$ by including correspondingly higher multiplets; however, the credibility of the non-relativistic harmonic oscillator may become questionable. These predictions will be interesting tests of our analysis.

Inclusion of both magnetic and electric interactions shows that the duality is non-trivial. Inasmuch as the magnetic terms dominate at large $Q^{2}$ in the quark model, duality can be realised for the dominantly transverse scattering of the deep inelastic region. For the longitudinal structure function, $F_{L}$, duality is again realised, with the breakdown into 56 and 70 as in Table 3:

Table 3: Relative Longitudinal Production Strengths, as in Table 2

\begin{tabular}{|c|c|c|c|c|c|c|}
\hline$S U(6):$ & {$\left[56,0^{+}\right]^{2} 8$} & {$\left[56,0^{+}\right]^{4} 10$} & {$\left[70,1^{-}\right]^{2} 8$} & {$\left[70,1^{-}\right]^{4} 8$} & {$\left[70,1^{-}\right]^{2} 10$} & total \\
\hline$F_{L}^{p}$ & 1 & 0 & 1 & 0 & 1 & 3 \\
\hline$F_{L}^{n}$ & 0 & 0 & 1 & 0 & 1 & 2 \\
\hline
\end{tabular}

However, for $F_{1}\left(Q^{2} \rightarrow 0\right)$ both electric and magnetic multipoles contribute and interfere with phases determined by the $J^{P}$ and the spin- $L_{z}$ correlations in the various $\mathbf{5 6}$ and $\mathbf{7 0}$ states. This causes dramatic $Q^{2}$ dependence 
in polarisation asymmetries [6, $6,0.9$ and enables the connection to the DrellHearn-Gerasimov sum rule at $Q^{2}=0$. Thus we predict that Bloom-Gilman duality must fail at $Q^{2}$ where the electric and magnetic multipoles have comparable strengths. Calculations in simplistic models successfully predicted that this would be at $Q^{2} \sim 0.5 \mathrm{GeV}^{2}[9]$; these results now merit, and are receiving, more detailed examination [i $[10]$. The data from Ref. [3i] show that even as low as $Q^{2}=0.5(\mathrm{GeV} / \mathrm{c})^{2}$ the integrated strengths of spectra at fixed $Q^{2}$ are within $10 \%$ of the corresponding integrals over the scaling curve. It will be interesting to verify the predicted breakdown at lower $Q^{2}$ and also to test if duality in the magnetic multipoles holds all the way to $Q^{2}=0$.

\section{A Possible Extension to Fragmentation Functions}

Our conclusion that the destructive interference between hadronic states of different symmetries is a critical feature of duality, can be applied to semi-inclusive hadroproduction, such as $\gamma(q) N \rightarrow \pi+X$. In the quark parton model in the ideal valence quark region when $u(x)=2 d(x)$ and at large $\mathrm{z}$, where $D_{u}^{\pi^{-}} / D_{u}^{\pi^{+}} \rightarrow 0$, it is trivial to obtain relations such as $F\left(\gamma p \rightarrow \pi^{+}+X\right) / F\left(\gamma p \rightarrow \pi^{-}+X\right)=8\left(=1 / 2\right.$ for neutron targets) [i] [1] $i_{1}$. The coherent picture described above can be applied to these processes. We find that destructive interference leads to factorisation and to duality, with for example

$$
F\left(\gamma p \rightarrow \pi^{+}+X\right) \equiv \sum_{\left(W^{\prime}=\mathbf{5 6}, \mathbf{7 0}\right)} F\left(\gamma p \rightarrow \pi^{+} W^{\prime}\right)
$$

where

$$
F\left(\gamma N \rightarrow \pi^{+} W^{\prime}\right)=\sum_{\left(N^{*}, N^{* *}\right)} F_{\left(\gamma N \rightarrow N^{*}\right)} D_{\left(N^{*} \rightarrow N^{\prime *} \pi\right)} \sim \sum_{q} e_{q}^{2} q(x) D_{q \rightarrow \pi}(z)
$$

where $D_{q \rightarrow \pi}$ is the quark $\rightarrow \pi$ fragmentation function, $F_{\left(\gamma N \rightarrow N^{*}\right)}$ is the $\gamma N \rightarrow$ $N^{*}$ transition form factor, and $D_{\left(N^{*} \rightarrow N^{\prime *} \pi\right)}$ is a function representing the decay $N^{*} \rightarrow N^{\prime *} \pi$ where $W^{\prime}$ is the invariant mass of the final state $N^{\prime *}$. The 
breakdown of $F\left(\gamma N \rightarrow \pi W^{\prime}\right)$ into the individual states in the supermultiplets for the final $W^{\prime}$ states is shown in Table 4 :

Table 4: $S U(6)$ and $S U(3) \times S U(2)$ Multiplet Contributions to Inclusive $\pi^{ \pm}$Photproduction

\begin{tabular}{lcccc}
\hline$W^{\prime}$ & $\gamma p \rightarrow \pi^{+}+W^{\prime}$ & $\gamma p \rightarrow \pi^{-}+W^{\prime}$ & $\gamma n \rightarrow \pi^{+}+W^{\prime}$ & $\gamma n \rightarrow \pi^{-}+W^{\prime}$ \\
\hline $\mathbf{5 6} ; \mathbf{8}$ & 100 & 0 & 0 & 25 \\
$\mathbf{5 6} ; \mathbf{1 0}$ & 32 & 24 & 96 & 8 \\
\hline $\mathbf{7 0} ;{ }^{\mathbf{2}} \mathbf{8}$ & 64 & 0 & 0 & 16 \\
$\mathbf{7 0} ; \mathbf{8}$ & 16 & 0 & 0 & 4 \\
$\mathbf{7 0} ; \mathbf{1 0}$ & 4 & 3 & 12 & 1 \\
\hline TOTAL & 216 & 27 & 108 & 54
\end{tabular}

Note the self-consistency of the results: $F\left(\gamma p \rightarrow\left(\pi^{+}+\pi^{-}\right)\right) / F\left(\gamma n \rightarrow\left(\pi^{+}+\right.\right.$ $\left.\pi^{-}\right)$) recovers the $3 / 2$ ratio familiar for the "total" $F^{p} / F^{n}$. We see also that duality may be obtained at large $Q^{2}, W^{2}$ when $W^{\prime}$ is integrated over the range up to $1.7 \mathrm{GeV}(\mathbf{5 6}+\mathbf{7 0})$. We see also that to a good approximation it may ensue for the $\mathbf{5 6}$ alone when Born terms and the $\Delta$ are included. To the extent that this is true, one may expect factorisation and approximate duality at small $Q^{2}, W^{\prime 2} \leq 3 \mathrm{GeV}^{2}$. A possible example of this was noticed long ago in Ref [i] $\left[\begin{array}{l}1 \\ 1\end{array}\right]$ for $\gamma p(n) \rightarrow \pi \Delta$ when $0.2 \leq|t| \leq 1 \mathrm{GeV}^{2}$.

In $\gamma N \rightarrow \pi+W^{\prime}$, the above Table strictly applies only to the imaginary part of the amplitudes. One should in principle also consider $u$-channel diagrams, where the $\pi$ is emitted prior to the photoabsorbtion. These diagrams would give an inversion of the ratios for $\pi^{+} / \pi^{-}$. Ref. [i] 1 has shown, via fixed $t$ dispersion relations, that at least for small $Q^{2}$, the $s$ and $u$-channel resonances tend to cancel in the real part, whereby the charge ratios are preserved. There is some indication from data on photoproduction that this is the case empirically $\left[1, \overline{1}, \overline{3_{1}}, 1, \overline{1} 2\right]$, but the dependence of these processes on $Q^{2}$ needs to be checked in explicit models and the precision of these ratios tested with data from CEBAF. We hope to return to these questions elsewhere.

Finally, we comment on a characteristic feature of one particle inclusive data: the production of jets. In this resonance duality approach, for any 
individual partial wave $L$ there is a specified angular distribution. Upon summing over $L$, the jet characteristics of the quark parton model arise as a result of the constructive interference of spherical harmonics in the near forward and destructive interference in the backward hemisphere [i1 $\left.{ }_{1}^{1}\right]$. As may be anticipated from the uncertainty principle, after summing over $L$ one expects an angular spread of the jet given by $\Delta \phi \sim h / L_{\max }$.

In this letter we have outlined the general features of a programme to understand not only duality, but also to define the "semi-local" averaging procedures that must be employed to see duality at low energies. We shall report elsewhere on detailed predictions that may be tested in the forthcoming experimental programs for CEBAF at Jefferson Laboratory. If we are successful in defining these averaging procedures, it would have a great impact on our ability to measure structure functions in kinematic regions that hitherto have been believed to be inaccessible.

\section{Acknowledgements}

We are indebted to W. Melnitchouk, and also to experimentalists at Jefferson Laboratory for discussions of their data. FEC is indebted to Jefferson Laboratory for hospitality and support. This work is supported, in part, by the European Community Human Mobility Program Eurodafne, contract NCT98-0169 and by DOE contract DE-AC05-84ER40150 under which the Southeastern Universities Research Associates (SURA) operates the Thomas Jefferson National Accelerator Facility (Jefferson Lab). 


\section{References}

[1] E.D.Bloom and F.J.Gilman, Phys.Rev. D4, 2901, (1971).

[2] A. de Rujula, H.Georgi and H.D.Politzer, Ann.Phys. 103 ,315, (1975);

X.Ji and P.Unrau, Phys. Rev. D52, 72 (1995).

[3] I.Niculescu et al., Phys. Rev. Letters. 85 1182, 1186 (2000).

[4] S.Jeschonnek, N.Isgur, W.Melnitchouk and J.W.Van Orden, "QuarkHadron Duality in Structure Functions", JLAB-THY-01-12, (in preparation).

[5] H. Harari Phys.Rev.Letters 201395 (1968);

P. G. O. Freund Phys. Rev. Letters 20235 (1968).

[6] F.E.Close, F.J.Gilman and I.Karliner, Phys Rev D6 , 2533, (1972);

F.E.Close and F.J.Gilman, Phys. Rev.D7 2258 (1973);

L.A.Copley, G.Karl and E.Obryk, Phys. Rev. D4, 2844 (1971).

[7] F.E.Close Phys.Letters 43 B 422 (1973).

[8] F.E.Close, Nucl Phys B80 269 (1974);

F.E.Close, Introduction to Quarks and Partons, chap 13 (1978);

N.Isgur, Phys Rev D59, 034013 (1999).

[9] F.E.Close and F.J.Gilman, Physics Letters 38B 541 (1972).

[10] For recent analysis, see the contribution in "Proceedings of $N^{*} 2000$ ", ed. V.Burkert (World Scientific, Singapore, 2001).

[11] F.E.Close, Introduction to Quarks and Partons, Academic Press (1978).

[12] I.Barbour, W.Malone and R.G.Moorhouse, Phys Rev. D4 1521 (1971).

[13] A.M.Boyarski et al Phys Rev. Letters 20300 (1968).

[14] N.Isgur and S.Jeschonnek, unpublished. 